\title{
INTERVIEW
}

\section{A Perspective on Natural Language Understanding Capability: An Interview with Sam Bowman}

Vasant Dhar ${ }^{1}$ and Sam Bowman ${ }^{2}$

Prof. Vasant Dhar, (Editor-in-Chief, Big Data): Sam, can you broadly classify the approaches we have taken in the last 30-40 years to understand natural language? What have been our experiences? What has worked? What has not worked?

Prof. Bowman: I can only give a very high-level overview of the earlier part of this history, but there are three broad families of approaches to language understanding in natural language processing (NLP).

From the 1960s through right around 1990, the predominant mode of research was rule and template based and very strictly symbolic. This kind of work on language understanding involved quite a lot of knowledge engineering, of trying to build systems around expert-designed representations for the semantics of various kinds of situations and the meanings of words.

The CYC project seems like a good example of the kind of work that was at the center of a lot of this type of thinking. This was an attempt to build a symbolic knowledge base of sort of all of human commonsense knowledge, such that when you hear a new sentence and you want to build some sort of representation of it, you can reference it against a large knowledge base. The word "dog" (i.e., the symbol "dog") has attached to it all of this information about what dogs are and what they do. It allows you to ground your meaning of symbols around such representations.

Prof. Dhar: Was this approach based on expediency, or was it based largely on how we thought we processed and understood language?

Prof. Bowman: I don't know how seriously people took the idea that this kind of work was cognitively plausible, but I think the predominant attitude was this was moving in the direction of strong artificial in- telligence (AI) - that you could solve language understanding in this way.

Prof. Dhar: And by strong AI, you mean what?

Prof. Bowman: Something that - at least in its ability to understand and manipulate language-is substantially comparable to a typical speaker. This is the classic, wildly ambitious goal of AI research.

Prof. Dhar: Okay, so that was the first approach. What about the other two?

Prof. Bowman: So starting in the early 1990s and continuing more or less through today is statistical NLP. This was a major transition driven simultaneously by two developments. The first was the increasing sense of despair about these handcrafted models-people coming to terms with the fact that the kinds of things that people talk about in language are too complex and too diverse to write down in any moderate-sized document or moderate-sized piece of code, and the amount of information that you would need to build a system by hand was just far more than you could tackle, even with very, very large-scale projects.

Second, getting access to reasonably large amounts of language data was becoming viable, and so the sort of predominant mode of work in this period shifted toward statistical machine learning. A lot of research was centered on designing probabilistic models, often generative models and graphical models that gave some intuition about how you would expect a machine to solve some language understanding tasks.

For translation, for example, this might involve learning in a purely statistical way what words in a French sentence tend to correspond to what words in the English translation of that French sentence and storing those 
frequent correspondences, and additionally in a purely statistical way trying to detect regularities in ways that word order might change from a sentence of French to a sentence of English, and then using all this information that has been inferred from data to run a system.

Prof. Dhar: So was this a hybrid consisting of bottom-up representations of correspondences and then finessing these with rules to figure out things such as word order?

Prof. Bowman: Somewhat, this style of machine translation system did incorporate the notion of both rules and word-level correspondences, but generally the vast bulk of the system would be inferred from data. You would define "the shape" of the model and what types of transformations you allow and then all of the work of actually fitting it to a particular pair of languages would be done from the data.

One example of how you might do translation under this approach would be first to take your French sentence and parse it-have another statistical model give you this kind of explicit tree structure that tells you how the French sentence fits together grammatically. Then you might use a bilingual dictionary that you have learned statistically to replace the French words with English words. And then you would use a bilingual grammar that matches grammar rules in French to grammar rules in English that tells you, "Okay, adjective-noun in one language translates to noun-adjective in the other language." And you would apply those rules to then transform the shape of the sentence and wind up with something that looks like a proper English translation.

Generally, every step of the way here is going to be probabilistic, and you are going to have some kind of distribution over many possibilities. And often you will have systems that have two parts where one part is producing candidate translations and another part is scoring for things such as whether they look like coherent English.

\section{Prof. Dhar: I guess now we have strayed into the third strand.}

Prof. Bowman: Getting closer. So the third broad approach is deep learning or artificial neural networks. You can debate whether this is really a truly separate strand or just a continuation of trends in statistical NLP. But I think there was a fairly sharp discontinuity in what kind of research people were doing as deep learning entered the field as the predominant mode of research.

\section{Prof. Dhar: What do you mean by a sharp disconti-} nuity? In what sense?

Prof. Bowman: With statistical NLP, there is generally a fundamental assumption that you, as the expert, are going to design the procedure for solving the problem, for example the procedure for doing translation, but that you will leave details to be filled in from data. The deep-learning world view is centered on building models that are capable of accepting an input of the right type, doing some arbitrary computation, and producing an output of the right type, and you don't even go as far as specifying what kinds of procedures or what kind of intermediate representations you think should be appropriate.

So for translation, for example, with the deep neural network space approach, you need not explicitly model the grammar of either sentence or the idea that one particular word of one language might explicitly correspond to another word of another language. You just build a model that reads strings and produces strings and learns everything in the middle from the data.

Deep learning in NLP is quite new, but neither of these two older strands has entirely died out. Deep learning was slower to come into NLP than into speech recognition or vision. It has only been around for maybe three or four years in the mainstream. But I would say deep learning is definitely a solid majority of ongoing research, if not a large majority. Statistical NLP is still a very active research area, though, and rule-based or schema-based NLP is around as a useful tool, especially applied system building, but it is not as active in ongoing research.

\section{Prof. Dhar: So where does Google Translate fall? The third approach?}

Prof. Bowman: Yes. Very solidly the third approach. There is great article in a The New York Times Magazine from December on the redesign of Translate. ${ }^{1}$ It is some of the best popular science writing I have seen on NLP.

A lot of the really prominent work on applying deep learning to NLP has come out of Google, and their big target application area was scaling up translation both in terms of quality and in terms of the number of languages they are able to handle.

Prof. Dhar: You mentioned a redesign? What was it before this redesign? How did it work?

Prof. Bowman: It fell under the statistical NLP approach. 
Prof. Dhar: So what gives the research community this optimism that something as simple as a deep net architecture can actually capture all these rules and subtleties of language? What is the basis for the optimism that you put a bunch of symbols in and the right symbols come out on the other side?

Prof. Bowman: I would not go as far as saying there is all that much optimism. I think a lot of people in the NLP community are very deeply interested in what the right representations for language are and how these systems should work internally. So people are disappointed, in a way, to see that these really opaque neural network models are working so well.

Prof. Dhar: When you say the right representations, what is a right representation for language?

Prof. Bowman: A lot of people in NLP are very interested in discovering a specific, clear, interpretable procedure for going from a string of symbols to something that looks more like a meaning or something that allows you to act as if you understand the sentence. And with deep-learning models, all of that is opaque.

Prof. Dhar: The intermediate representations do not actually correspond to something that resonates with you?

Prof. Bowman: Yes. A lot of people are sort of being dragged kicking and screaming into these research methods because empirically they have turned out to work extraordinarily well, and there is also a lot of interest in reverse engineering them and trying to understand what systems such as this are learning. That is still in its infancy though.

But I guess to get to your original question, I think this is all about empirical results-that in machine translation, for example, progress had really slowed down, that it would take quite a lot of work, subtly modifying very complex systems, to make any kind of progress in translation quality, and the introduction of these neural network-based methods dramatically changed what was possible.

Prof. Dhar: Without sort of getting into any claims of cognitive plausibility, it is just, "Hey, this stuff seems to work."

Prof. Bowman: Yeah, exactly.
Prof. Dhar: Indeed, a fair amount of research in deep learning these days is about why these things actually work as well as they do.

Prof. Bowman: Yeah. There is not a lot of consensus on it.

Prof. Dhar: So what are we struggling with at the moment? What is really hard to do with these newer architectures?

Prof. Bowman: On a sort of immediate and practical front, these kinds of models depend completely on large, annotated data sets. Doing high-quality translation with these models is only possible because so much translation data get produced in the course of business for lots of organizations, and it is possible to collect potentially hundreds of millions of pairs of translated sentences. And that is what makes it possible to train models like this.

If you are trying to solve all image understanding problems where you only have a few examples of the task, where you do not have this kind of massive labeled data set, we still do not really know even where to begin.

Prof. Dhar: So this may be a bit of a tangent, but let me bring it in anyway. In the 1980s and 1990s when people were trying to build expert systems such as in medical diagnosis, they were trying to make the interfaces as "natural" as possible. And their goals were pretty ambitious in that they were actually trying to model the entire dialogue between the human and machine. I don't think that got very far.

It seems to me that what we have now with these newer approaches is the ability to go from one string to another and do a pretty good job of translating French to English and English to French. But I guess we have not really made much of a dent in solving the problem of facilitating coherent discourse yet. Is that right?

Prof. Bowman: Yeah. Yeah.

Prof. Dhar: Is that something people are looking at in the machine learning community at all, or are people more focused on the simpler units of analysis?

Prof. Bowman: I think that ties into two other big open issues that I see. These neural network models are not very good at high-level planning, and they are not very good at producing paragraphs or documents 
of text or long sequences of turns in a conversation that have a structure. The more long-term planning you want the model to have, the more data you are going to need. So for something such as generating coherent documents, these models simply cannot be trained with the kind of data that we have access to. That is a major problem. I think that there is a lot of room for creativity here in the details of model design.

Prof. Dhar: By the way, is that similar to the difficulty of writing a screenplay where you need this sort of coherence and interplay between linear and nonlinear structure, and there is sort of a craft to that.

Prof. Bowman: Yeah. If you run a neural network chatbot, and chatbots by some measures are something that we are very good at building, I think they represent a lot of the strong points of modern NLP technology. But nonetheless, if you actually interact with one, you might see that it can hold what looks like a conversation for a couple of turns, but that it clearly has no understanding of the relevance of anything that was said more than a few sentences ago. So, from the perspective of simple language understanding, we are very, very rapidly improving in our ability to understand what a sentence says on its face, what its literal meaning is, and what its obvious consequences are. These models can also be good at understanding nonstandard speech such as slang or strange usage, but a lot of language understanding and a lot of conversation goes beyond just understanding this kind of...

\section{Prof. Dhar: Sentences in isolation?}

Prof. Bowman: Yeah. So the kinds of things that we are much less good at are things such as pragmatics. This is the skill of understanding what a sentence contributes to a dialogue or to a discourse by looking both at what was said and what was not said and how it relates to conversational context. This is something that we are doing constantly in every sentence in any conversation or exchange you would ever have.

So, pragmatic inference is something huge that we really just do not really have the tools to work with. I do not think this is necessarily something that demands more data, but our models are not designed to accommodate this kind of thinking-interpreting sentences with goals and communicative dynamics in mind.
And also closely related to that is commonsense knowledge. These systems might learn to understand language without necessarily learning that if you set a mug on a table, it will stay there, but if you set a balloon on a table, it might not.

This kind of background knowledge is not generally going to be written down anywhere. This is not going to be something that you can really learn directly from anything that is going to be included on the Internet or in databases, but you often do need to know this kind of knowledge in order to do pragmatic inference, in order to reason out the consequences of what someone is saying.

Prof. Dhar: Right. So, just two more things I wanted to touch on. Why is there all this excitement around "word embeddings" these days? What is that all about? Why does it work? When does it work, and when does it not work?

Prof. Bowman: Yeah. So word embeddings represent a set of related strategies for doing unsupervised learning on raw text. This is a way of trying to extract some kind of useful knowledge in a very fast, automatic way from, in some cases, the entire Internet. You can run these models on hundreds of billions of words of text in a relatively short amount of time.

And what you get when you run a word-embedding model is a dictionary with hundreds of thousands of words of some language such as English paired with these dense vector representations. And what these...

Prof. Dhar: Just to clarify, the dense vector representation is at a word level?

Prof. Bowman: Yeah. You might have typically something like a 300-dimensional vector paired with every word, and that is the embedding. You can picture these words as being points in a 300-dimensional Euclidian space.

The most crucial thing this gives you-and it took people in NLP some time to realize just how important this would wind up being-is a clear understanding of similarity of meaning between words. One thing these word-embedding methods are very good at extracting is that, for example, synonymous words are generally going to be very, very close to one another in this high-dimensional space.

Words that are in the same category, such as days of the week or grammatical articles or prepositions, words 
that form some kind of class, are going to tend to cluster together in space.

Prof. Dhar: I want to come back to what that means. But go on.

Prof. Bowman: But what having these representations of word meaning allows you to do is to train machinelearning models in NLP, things such as statistical models or deep-learning models, on moderate amounts of data where they might never see some words, and they might see some words only a very small number of times, but to allow them to generalize what they learn from that moderately sized data set to new data with new words.

You might, for example, be training a simple sentiment analysis system, where your goal is to look at a sentence and judge whether it is expressing a positive or a negative attitude toward the thing that it is describing.

And maybe you have seen the sentence, "This is an excellent movie," and you know that a sentence such as that is going to express a very positive sentiment.

Maybe you have never seen the word "awesome" before, or maybe you have seen it but in a mix of contexts that does not really make it clear whether it is going to be giving you positive or negative sentiment. Now you encounter the sentence, "This is an awesome movie."

If you represent these words inside the model using these pre-trained vectors, what this embedding space is going to tell you is that whatever you do in response to seeing the word "excellent," you should do roughly the same thing in response to seeing the word "awesome," and that by treating similar words similarly, you can encourage models to generalize smoothly to sentences that might have a similar structure to something you have seen before but which is different in its precise wording.

This kind of winds up being like this sort of Sriracha of NLP; it is not really fundamental to solving any problem, but for almost any problem in language understanding, it gives you a substantial boost in performance. You can do noticeably better by using word embeddings than you can do without.

Prof. Dhar: So if you incorporate word embeddings as part of an understanding system, it gives you the flexibility to interpret language in a more general way?
Prof. Bowman: Yeah. And I think there was a lot of scientific excitement around these. The idea has been around for at least 20 years, and it has been kind of bubbling in the background in the NLP community. But some really high-profile successes came out in 2013, again out of Google, and in the next major NLP conference about six months later, suddenly almost half of the papers in the conference were about either ways to use word embeddings or ways to understand word embeddings.

Prof. Dhar: So I want to come back to something you said earlier, which is that they "lie close." So how does that happen? They have got to be trained, I guess, for a system to learn that they are close?

Prof. Bowman: Yeah. So there are different approaches to training word embeddings from unlabeled data that work in fairly different ways, but they are all built on this idea about language understanding called distributional semantics. And the idea here is that the distribution of a word, essentially what contexts it shows up in, what kinds of other words it tends to show up near in a text, gives you a very strong cue to what that word means.

Prof. Dhar: And when you say the distribution of the word, you mean literally the other words it co-occurs with?

Prof. Bowman: Yes. So the kind of thing that a word embedding model might do is look at a sentence and take out one of the words and try to predict what the missing word is. So here you are essentially saying, "All right, given all of these context words, I want to try to fill in this blank," and it is going to be trying to do this in this simple geometric way using these vector representations of words.

By training them all on this task-and you can train the model on this task with almost infinite data because all you need are sentences-you can precisely tune word embeddings for this purpose.

What has turned out to be fortuitously the case is that word embeddings that allow you to solve this problem also give you a pretty broadly useful picture of meaning similarity and key axes of variation and meaning.

Prof. Dhar: So last question. Where do we stand in terms of sort of the state of the art in sentiment 
analysis? How far along are we at reading a story and summarizing what it is about? I mean not just positive/negative, but a more robust summary of the article?

Prof. Bowman: I see that that as getting at a couple of things. The definition of sentiment analysis I hear most often in NLP is just simple classification, positive/negative, usually on sentences or paragraphs.

In this kind of setting, I think we are most of the way there. This is exactly the kind of problem that deep neural networks and word embeddings makes very easy if you have enough data, and we do have a lot of data for this kind of problem. This problem is one that has a sort of blurry edge in terms of kind of real hard, serious language understanding, though, so we aren't going all the way there without some additional breakthroughs.

Prof. Dhar: So if I understand you right, you are saying that if it is a question of classification into some broad categories, we can do that pretty well. Going beyond that toward deeper understanding is much harder.

Prof. Bowman: Yeah. And I should say we are as close to solving that version of sentiment analysis as we are to solving anything in NLP where there is sort of a fuzzy border with real hard language understanding. Some examples of this problem can be subtle and depend on knowledge and word-modeling pragmatics and get into all of this hard stuff, but that is just a small piece of the pie.

The broader problem of reading something such as a news article and either summarizing it or, even worse, explaining how it is relevant to some other entity or situation is at the other end of the spectrum. That is touching on all of the biggest unsolved problems in NLP that everyone would like to be able to make progress. That is, combining this ability to really understand long narratives that are going to require you to develop some kind of picture of what is the world like given that I have learned all of these things from this article, and it is going to require you to sort of creatively generate text.

Prof. Dhar: It almost seems related to that earlier discussion we were having on discourse analysis.

Prof. Bowman: Yeah.
Prof. Dhar: That tying the context together across different sentences or paragraphs is extremely difficult.

Prof. Bowman: Yeah. I think to a first approximation, NLP problems where we have a lot of data that involve words and short phrases-where all of the action is at that scale-are ones that we can solve fairly easily. Tasks that involve understanding sentences or producing sentences-or that involve understanding or producing documents-that's still pretty far afield.

Prof. Dhar: Okay, good. One last thing. I want to talk about tools for researchers and for practitioners. Where do we stand with respect to tools? What kinds of tools are you using as a researcher? What kinds of tools should people in businesses be using who actually want to make a dent in terms of understanding unstructured data? What are the choices out there, and how should they be thinking about it?

Prof. Bowman: I might not be the best person to comment on some of these issues because I know there are some parts of the tool-building community that I am not as exposed to. But broadly right now, I see two big categories of software tools that are important to NLP but that handle it quite differently.

There are toolkits such as Stanford CoreNLP or NLTK, the natural language toolkit. Google has competitors to this as well. There are a few other toolkits in this neighborhood that provide you with lots of low-level functionality for working with things such as splitting text into words, doing very simple classification problems, maybe parsing out tree structure representations from text. They give you all these building blocks.

Those tend to be built up out of fairly mature technologies. They tend to be more based on statistical NLP or even rule-based methods, so you can get toolkits that represent effective, mature technologies for the simpler problems.

For building things on top of this or solving more complex problems, there is not a lot-there are not that many great tools out there that are all that specific to NLP. There are not excellent libraries for a lot of tasks that we would like to be able to solve, such as summarization, such as sentiment, that fit this middle ground between being just a black-box system, such as what IBM provides, that just sort of does something 
and a really low-level tool box where you have to do quite a lot of work yourself.

And I should say in terms of what researchers tend to spend a lot of their time using software-wise and what you see a lot in sort of more ambitious, more hands-on industrial applications are deep-learning libraries. And which of these software libraries is considered the fastest or the easiest to work with has been changing pretty quickly. You will get a new big contender roughly every nine months.

But this is where tools such as TensorFlow or Keras or Torch are relevant-those are the manuals that I spend my days reading in the process of really trying to build substantially new tools in NLP.
Prof. Dhar: That was very useful, Sam. It clarified a lot of things for me, and I think readers will find this very educational and pragmatic. Thank you so much for this service to the community!

\section{References}

1. Lewis-Kraus G. The great A.I. awakening. The New York Times Magazine, December 14, 2016. Available at: www.nytimes.com/2016/12/14/ magazine/the-great-ai-awakening.html?_r=2

Cite this article as: Dhar V, Bowman S (2017) A perspective on natural language understanding capability: An interview with Sam Bowman. Big Data 5:1, 5-11, DOI: 10.1089/big.2017.29016.sbo. 